\title{
Observed Abundance Features and their Implications for Chemical Evolution
}

\author{
Takuji Tsujimoto ${ }^{1,3}$ and Toshikazu Shigeyama ${ }^{2}$ \\ ${ }^{1}$ National Astronomical Observatory, 2-21-1 Osawa, Mitaka-shi, Tokyo 181-8588, Japan \\ ${ }^{2}$ Research Center for the Early Universe, University of Tokyo, Bunkyo-ku, Tokyo 113-0033, Japan \\ ${ }^{3}$ E-mail: taku.tsujimoto@nao.ac.jp
}

Received 2003 October 1, accepted 2004 January 19

\begin{abstract}
We describe the star formation histories of the Milky Way dwarf spheroidal galaxy and the globular cluster $\omega$ Centauri in terms of an inhomogeneous chemical evolution model developed for the Galactic halo. The observed abundance trends seen in neutron-capture elements together with $\alpha$-elements constrain our models to shed light on the histories of these nearby galaxies and $\omega$ Cen. The origin of low- $\alpha$ stars and a new picture of the globular cluster formation scenario induced by cloud-cloud collisions are also presented.
\end{abstract}

Keywords: globular clusters: general — globular clusters: individual ( $\omega$ Centauri) — stars: abundances — galaxies: dwarf — planetary systems

\section{Introduction}

There is no doubt that the recent progress on abundance determination for numerous solar neighbourhood stars promotes a better understanding of the nature of the Milky Way, because stellar abundance patterns bring us valuable information on how the Milky Way formed and has evolved (Wheeler, Sneden, \& Truran 1989; McWilliam 1997). In this section, we present a new chemical evolution model for the Galactic halo as well as an interpretation of the abundance features seen in the Galactic halo field stars.

In the usual approach (Tinsley 1980), stars are assumed to form from well-mixed gas at a rate proportional to some power of the gas density. However, the validity of using this assumption for halo stars has been challenged by the observed abundance patterns of very metal-poor stars (McWilliam et al. 1995; Ryan, Norris, \& Beers 1996), which are incompatible with those predicted from such a simple star formation rate. This problem has recently been overcome by a newly proposed model in which halo stars are always born from dense shells swept up by individual supernova remnants (SNRs) and necessarily retain the abundance patterns of heavy elements ejected from supernovae ( $\mathrm{SNe}$ ) of various progenitor masses (Shigeyama \& Tsujimoto 1998; Tsujimoto \& Shigeyama 1998; Tsujimoto, Shigeyama, \& Yoshii 1999, 2000).

This model was motivated by the fact that the mixing length of heavy elements ejected from $\mathrm{SNe}$ is too small to achieve chemical homogeneity over the entire halo. Current estimates of velocity dispersion and total mass of the stellar components in the Galactic halo indicate that consecutive star-forming processes are likely to have been confined in separate regions or clouds having mass scales of $M_{c} \approx 10^{6-7} \mathrm{M}_{\odot}$, which make up the entire baryonic halo. In a proto-galaxy, the metal-free Population III stars

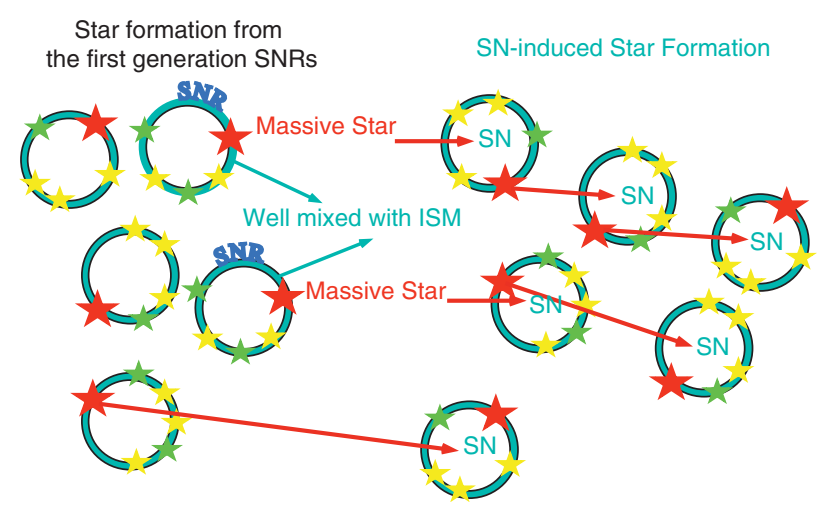

Figure 1 Schematic illustration of the SN-induced star formation.

of the first generation could have been born in highly compressed layers behind shock waves caused by supersonic initial turbulence. The massive ones of them eventually explode as metal-free $\mathrm{SNe}$, which trigger the formation of stars in dense shells of mass $M_{\mathrm{sh}}$. The mass $M_{\mathrm{sh}}$ is approximated by the formula (Shigeyama \& Tsujimoto 1998)

$$
\begin{aligned}
M_{\mathrm{sh}}= & 6.5 \times 10^{4} \mathrm{M}_{\odot}\left(\frac{E_{0}}{10^{51 \mathrm{ergs}}}\right)^{0.97} \\
& \times n_{1}^{-0.062}\left(\frac{\sigma_{v}}{10 \mathrm{~km} \mathrm{~s}^{-1}}\right)^{-9 / 7}
\end{aligned}
$$

where $E_{0}$ is the explosion energy of an SN, $n_{1}$ the density of an interstellar medium (ISM), and $\sigma_{v}$ the velocity dispersion of the ISM. This SN-induced formation of stars goes into generations, as schematically illustrated in Figure 1, and terminates when SNRs become unable to sweep up enough gas to form dense shells. 


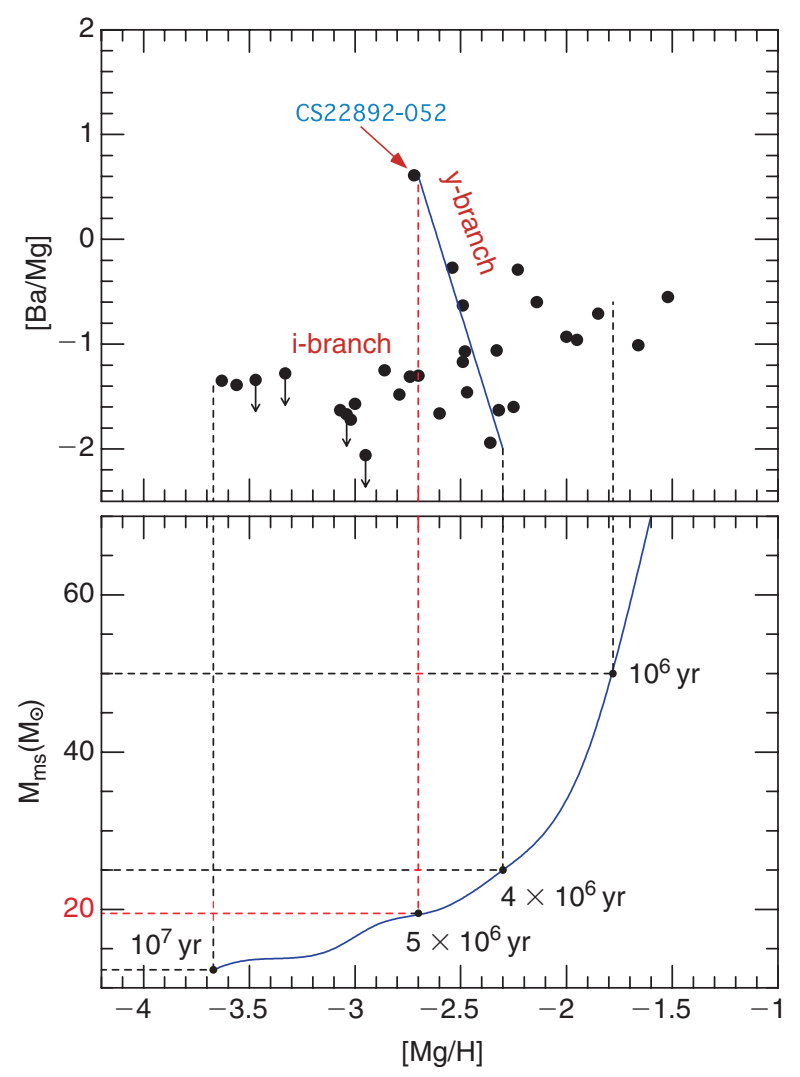

Figure 2 Upper panel: Correlation of $[\mathrm{Ba} / \mathrm{Mg}]$ with $[\mathrm{Mg} / \mathrm{H}]$ for metal-poor stars (McWilliam 1998). The thick line is the left-side boundary to the $y$-branch representing the mass range of the r-process site. McWilliam (1998) showed that the $[\mathrm{Ba} / \mathrm{Eu}]$ ratios for these stars are consistent with pure r-process ratios and concluded that $\mathrm{Ba}$ in extremely metal-poor stars is also of r-process origin. Lower panel: SN II progenitor mass $M_{\mathrm{ms}}$ plotted as a function of $[\mathrm{Mg} / \mathrm{H}]$ inside the shell swept up by the SNR. The stellar lifetimes are also shown for four reference masses of $M_{\mathrm{ms}}=12,20,25$, and $50 \mathrm{M}_{\odot}$. The adopted $\mathrm{Mg}$ yields and the stellar lifetimes are from Tsujimoto et al. (1995) and Timmes, Woosley, \& Weaver (1995), respectively. Dashed lines in the lower and upper panels illustrate how we assign $M_{\mathrm{ms}}$ to each star along the boundary.

In this picture, the abundance pattern of each star is composed not only of the heavy elements ejected from an SN of the preceding generation but also of those that have been already included in the ISM swept up by the SNR. However, very metal-poor halo stars might have inherited the abundance pattern of the ejecta of the preceding SN due to a negligible contribution from heavy elements in the ISM to their stellar abundances. From this viewpoint, a unique feature in the observed distribution of $[\mathrm{Ba} / \mathrm{Mg}]$ versus $[\mathrm{Mg} / \mathrm{H}]$ for very metal-poor stars can be adequately interpreted as shown in Figure 2. It should be, however, noted that the $\mathrm{Ba}$ abundances in metal-poor halo stars is subject to large uncertainties. We associate this feature, a bifurcation of the observed elemental ratios into two branches in the $\mathrm{Mg}$ abundance interval $-3.7 \leq[\mathrm{Mg} / \mathrm{H}] \leq-2.3$, with two distinct processes. The first branch, which we call the y-branch, is associated with the production of $\mathrm{Ba}$ and $\mathrm{Mg}$ from individual massive $\mathrm{SNe}$. We conclude that $\mathrm{SNe}$ II with
$M_{\mathrm{ms}} \approx 20 \mathrm{M}_{\odot}$ are the dominant source of r-process nucleosynthesis in the early Galaxy (Tsujimoto et al. 2000; Tsujimoto \& Shigeyama 2001). An SN-induced chemical evolution model with the $M_{\mathrm{ms}}$-dependent $\mathrm{Ba}$ yield creates the y-branch, reflecting the different nucleosynthesis yields of $[\mathrm{Ba} / \mathrm{Mg}]$ for each SN II with $M_{\mathrm{ms}} \gtrsim 20 \mathrm{M}_{\odot}$. The second branch, which we call the i-branch, is associated with the elemental abundance ratios of stars which were formed in the dense shells of the ISM swept up by $\mathrm{SNe}$ II with $M_{\mathrm{ms}}<20 \mathrm{M}_{\odot}$ that do not synthesise r-process elements, and applies to stars with observed $\mathrm{Mg}$ abundances in the range $[\mathrm{Mg} / \mathrm{H}]<-2.7$. The Ba abundances in these stars reflect those of the interstellar gas at the (later) time of their formation. The existence of a $[\mathrm{Ba} / \mathrm{Mg}]$ i-branch strongly suggests that $\mathrm{SNe}$ II which are associated with stars of progenitor mass $M_{\mathrm{ms}} \leq 20 \mathrm{M}_{\odot}$ are infertile sources for the production of r-process elements.

These findings together with the recent progress on abundance determination beyond the solar neighbourhood lead us to carry our study forward to the chemical evolutions for the Milky Way dwarf spheroidal (dSph) galaxies and the peculiar globular cluster $\omega$ Centauri.

\section{The Milky Way Dwarf Spheroidal Galaxies}

We have now reached the stage where we can learn the history of other nearby galaxies from detailed elemental abundances of individual stars. Remarkable features in the abundance patterns of dSph stars are found in neutron-capture elements such as $\mathrm{Ba}$ and $\mathrm{Eu}$, and also in $\alpha$-elements. Using these elemental abundance features, we discuss the history of $\mathrm{dSph}$ galaxies and the external pollution onto the surface of stars triggered by engulfment of planets.

\subsection{History of dSph Galaxies}

Stellar abundance pattern of n-capture elements is used as a powerful tool to infer how the star formation proceeded in $\mathrm{dSph}$ galaxies. It is found that the abundance correlation of $\mathrm{Ba}$ with $\mathrm{Fe}$ in stars belonging to $\mathrm{dSph}$ galaxies orbiting the Milky Way, i.e. Draco, Sextans, and Ursa Minor have a feature similar to that in Galactic metal-poor stars. Figure 3 shows the correlation of $[\mathrm{Ba} / \mathrm{Fe}]$ with $[\mathrm{Fe} / \mathrm{H}]$ for $\mathrm{dSph}$ stars (Shetrone et al. 2001), together with that for Galactic metal-poor stars ( $\times$; McWilliam 1998). Filled circles represent dSph stars with $[\mathrm{Fe} / \mathrm{H}]$ shifted by $\Delta[\mathrm{Fe} / \mathrm{H}]=-0.6$ dex. An excellent coincidence between two distributions appears: both metal-poor stars and dSph stars populate two separate branches in the $[\mathrm{Ba} / \mathrm{Fe}]-[\mathrm{Fe} / \mathrm{H}]$ plane, i.e., the first $y$-branch in which stars have positive $[\mathrm{Ba} / \mathrm{Fe}]$ ratios (i.e., greater than the solar ratio), and the second i-branch with the upper bound at $[\mathrm{Ba} / \mathrm{Fe}]=-1$. This feature can be reproduced by our inhomogeneous chemical evolution model as already described in the previous Section.

The metallicities of metal-poor stars are determined by two factors: what mass of heavy elements a SN supplies and what mass of ISM was eventually swept by a single 


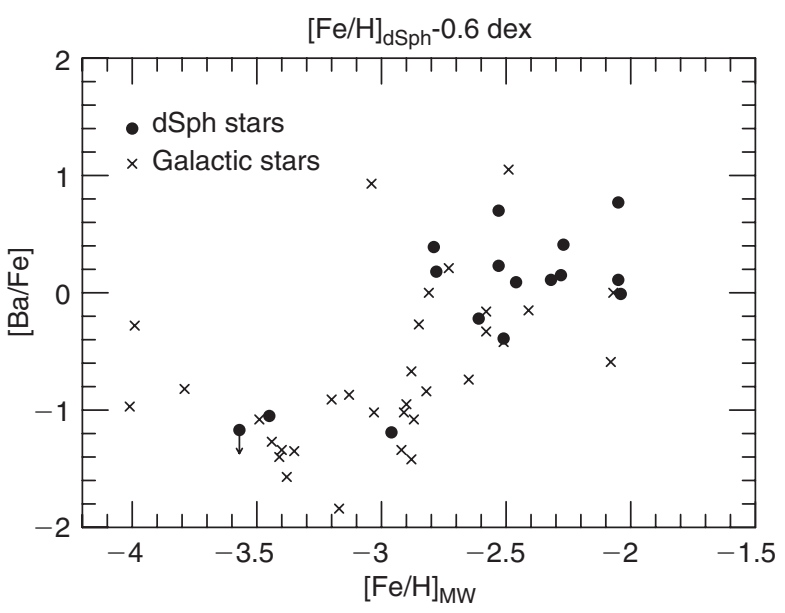

Figure 3 Correlations of $[\mathrm{Ba} / \mathrm{Fe}]$ with $[\mathrm{Fe} / \mathrm{H}]$ for $\mathrm{dSph}$ stars (• Shetrone et al. 2001) and Galactic halo field stars (McWilliam 1998). The data of dSph stars are shifted by the amount of $\Delta[\mathrm{Fe} / \mathrm{H}]=-0.6 \mathrm{dex}$ (see the text). The K-star of Ursa Minor is excluded from the plots because it is recognised to be a carbon star, most probably self-enriched by s-process material.

SN explosion (Shigeyama \& Tsujimoto 1998). The former is determined exclusively by each SN. On the other hand, the latter quantity is influenced by the environment such as the velocity dispersion $\sigma_{v}$ and density $n_{1}$ of the ISM. If SNe in the Milky Way and those in dSph galaxies are similar, different environments should give rise to $\Delta[\mathrm{Fe} / \mathrm{H}]$, discussed above. Since the mass $M_{\text {sh }}$ swept up by an $\mathrm{SN}$ is much more sensitive to the velocity dispersion than to the density; $M_{\mathrm{sh}} \propto \sigma_{v}^{-9 / 7} n_{1}^{-0.062}$ (see Eq. (1)), it is likely that a larger velocity dispersion in $\mathrm{dSph}$ galaxies at the star formation epoch enhanced the stellar $[\mathrm{Fe} / \mathrm{H}]$. Quantitatively, $\Delta[\mathrm{Fe} / \mathrm{H}]=-0.6$ dex corresponds to a $\sigma_{v} \sim 26 \mathrm{~km} \mathrm{~s}^{-1}$. The velocity dispersion of stars must have a similar value in their equilibrium configuration. The present value of $\sigma_{\star}$ value measured in Galactic dSph galaxies are about $9 \mathrm{~km} \mathrm{~s}^{-1}$. These two significantly different velocity dispersions can be connected in the context of a galaxy losing mass by the tidal force of the Milky Way. As a result, the total mass of each dSph galaxy is found to have been originally $\sim 25$ times larger than at present, using the relation $\sigma_{\star} \propto M_{\mathrm{dSph}}^{1 / 3}$.

Then the history of Galactic dSph galaxies that we propose is as follows. The proto-dSph galaxy consisted of gas and dark matter, and was approximately 25 times as massive as a present $\mathrm{dSph}$ galaxy. Star formation lasted for about 200 Myr; meanwhile, 5\% of the gas converted into stars, which has been estimated from the chemical evolution model for $\mathrm{dSph}$ galaxies (Tsujimoto \& Shigeyama 2002). Afterward, the remaining gas was dispelled by the last SN explosions. On the other hand, dark matter which surrounds stars has lost more than $90 \%$ of its mass through the tidal interaction with the Milky Way.

\subsection{The Origin of Low $[\alpha / F e]$ Ratios in SSph Galaxies}

A puzzling abundance feature has been seen in the $[\alpha / \mathrm{Fe}]$ ratios for dSph stars. Shetrone et al. $(2001,2003)$ found

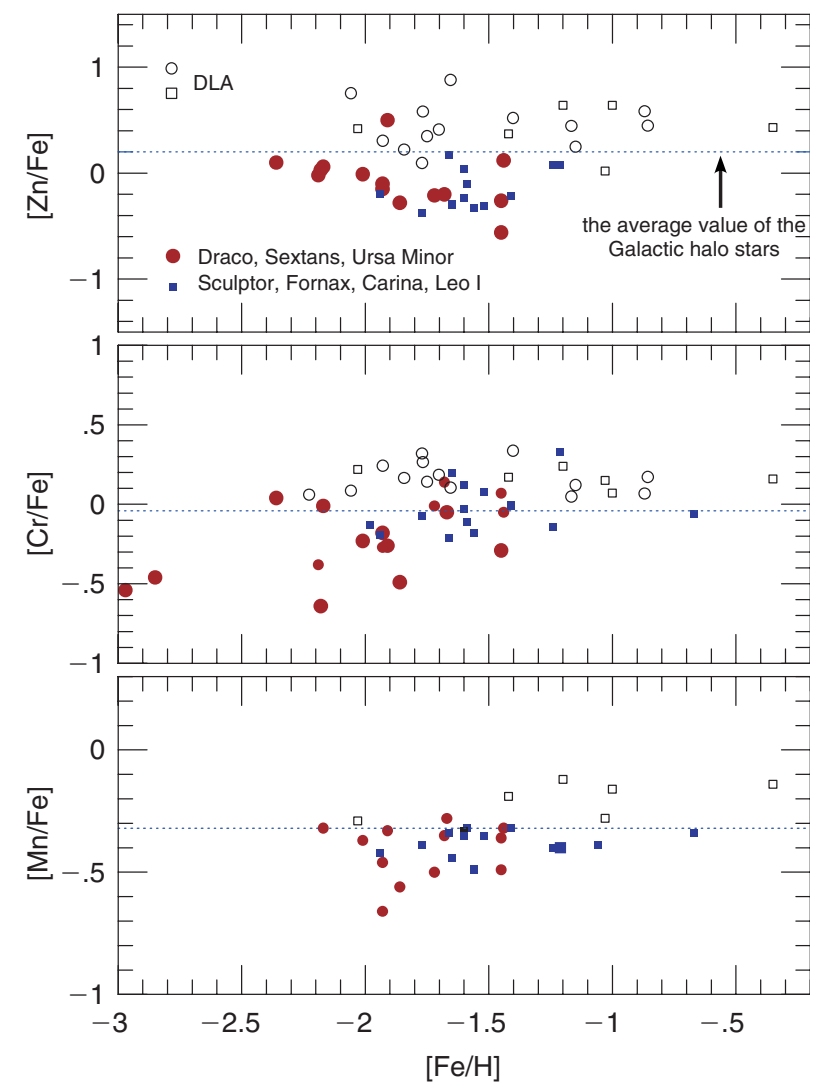

Figure 4 The ratios of Fe-peak elements to Fe for dSph stars $(\bullet,-\bullet)$ together with those for DLA (o Prochaska \& Wolfe 2002; $\square$ Pettini et al. 2000). The dashed lines represent the average $[\mathrm{X} / \mathrm{Fe}]$ ratios of the Galactic halo stars.

that $\mathrm{dSph}$ stars exhibit lower $[\alpha / \mathrm{Fe}]$ ratios than stars in the Galactic halo. A likely interpretation of these observational results is that $\mathrm{dSph}$ galaxies experienced different enrichment by $\mathrm{SNe}$ from the Galactic halo (e.g. Shetrone et al. 2003). It has two possibilities. One is a contribution from $\mathrm{SNe}$ Ia in dSph galaxies. However, abundance ratios among n-capture elements for most of dSph stars exhibit nearly pure r-process ratios (Tsujimoto \& Shigeyama 2003a). Taking into account a longer lifetime of SN Ia progenitors than s-process enrichment, the possibility that a large amount of Fe from SNe Ia lowered the $[\alpha / \mathrm{Fe}]$ ratios is unlikely. Another possibility is different yields from $\mathrm{SNe}$ II, likely due to a different initial mass function (IMF) in $\mathrm{dSph}$ galaxies from Salpeter IMF. However, there is no signature in other elemental ratios such as $[\mathrm{Mn} / \mathrm{Fe}]$. It is therefore also unlikely.

Observed abundance patterns for dSph stars in comparison with those for damped Lyman- $\alpha$ absorbers (DLAs) shed light on this subject. As shown in Figure 4, abundance ratios for $\mathrm{dSph}$ stars are distributed over the opposite side of DLAs with average values for Galactic halo stars in the middle. If we interpret abundance trends of DLA as a result of dust depletion, abundance patterns for $\mathrm{dSph}$ stars might turn out to reflect those for dust grains, which implies that accretion of dust grains onto the surface of $\mathrm{dSph}$ stars should have occurred. Then what is the nature of the dust grains? Here we conclude that it should be 
planets, paying attention to the fact that in $\mathrm{dSph}$ galaxies we have observed very luminous red giants corresponding to a very late stage in the red giant branch (RGB).

The envelope of a star expands as it evolves along the RGB, and finally the size reaches up to a few AU. Therefore if a star harbours planets, some of them will be eventually engulfed by their host star during the late stage of the RGB. For instance, in our solar system, the Sun is expected to eventually engulf all the terrestrial planets, although the gas giant planets will be beyond the reach of the evolved Sun. The ongoing hunting of extra-solar planets has revealed that there are some planetary systems in which massive planets orbit their host stars with the semimajor axes of the order of only 1 AU. The average mass and semi-major axis of the planet orbits are found to be 3 $M_{J}$ and 1.2 AU in a sample of 107 planets in 93 planetary systems. ${ }^{1}$ This leads to an implication that some fractions of stars with planets will be likely to engulf giant planets till the stars evolve to the tips of the RGBs. Though the mass of the dense core in a gas giant planet such as Jupiter and Saturn in our planetary system is uncertain, a large amount of $\mathrm{Fe}$ is expected to be contained in their cores, compared with terrestrial planets like Earth (Guillot 1999).

There is no information on which fraction of each element was depleted from the gas phase in the protoplanetary gas disks around metal-poor stars with the metallicity range of $-2 \lesssim[\mathrm{Fe} / \mathrm{H}] \lesssim-1$. A recent observation of the ISM in the small magellanic cloud with the average metallicity of $[\mathrm{Zn} / \mathrm{H}] \sim-1$ might, however, shed light on this issue (Welty et al. 2001); Fe-group elements are depleted as much as in the Galactic ISM, whereas $\alpha$-elements such as $\mathrm{Mg}$ and $\mathrm{Si}$ are nearly undepleted, though Si has a higher condensation temperature than Fe. This depletion pattern would support the scenario in which low- $\alpha$ dSph stars engulfed giant planets.

\section{$3 \omega$ Centauri}

The potential history and characteristics of the globular cluster (GC) $\omega$ Centauri remains largely unresolved, although its most conspicuous features can be seen in the elemental abundance properties. Most prominently, there exists a large dispersion in metallicity $(-2 \lesssim[\mathrm{Fe} / \mathrm{H}] \lesssim-0.5)$ among its member stars (e.g., Norris \& Da Costa 1995; Suntzeff \& Kraft 1996) in contrast to other Galactic GCs which exhibit essentially no dispersion. In addition, this GC contains stars that display a significant enhancement of s-process elements relative to Fe and r-process elements (Norris \& Da Costa 1995).

Regarding the latter feature, a study by Smith et al. (2000) identified two distinct populations in terms of correlation between $[\mathrm{Ba} / \mathrm{Eu}]$ and $[\mathrm{Fe} / \mathrm{H}]$ for $10 \omega$ Cen stars $(\bullet$ in Figure 5). One population has metallicity in the range of $[\mathrm{Fe} / \mathrm{H}]<-1.8$ and low $[\mathrm{Ba} / \mathrm{Eu}]$ ratios, corresponding closely to the pure r-process origin ratio, and the other population has $[\mathrm{Fe} / \mathrm{H}]>-1.6$ with high $[\mathrm{Ba} / \mathrm{Eu}]$ ratios, ascribed to a large s-process nucleosynthesis contribution.

\footnotetext{
${ }^{1}$ http: / / www . obspm. fr/encycl / catalog.html
}

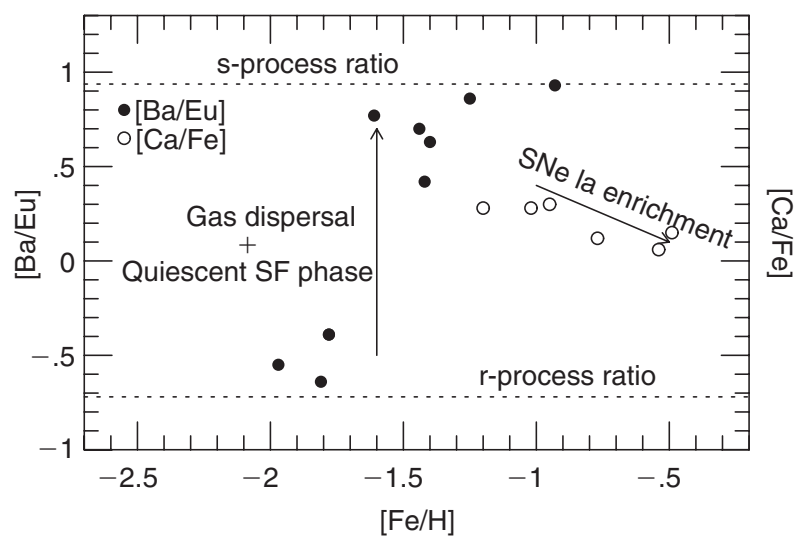

Figure 5 Correlations of $[\mathrm{Ba} / \mathrm{Eu}](\bullet$ Smith et al. 2000) and $[\mathrm{Ca} / \mathrm{Fe}]$ (o Pancino et al. 2002) with $[\mathrm{Fe} / \mathrm{H}]$ for $\omega$ Cen stars. The dashed lines show the $[\mathrm{Ba} / \mathrm{Eu}]$ ratios theoretically anticipated from the r-process origin and s-process nucleosynthesis. Some comments are also attached.

Another unique feature of the elemental abundance can be observed in the abundance distribution function (ADF) of $\omega$ Cen stars (Norris, Freeman, \& Mighell 1996; Suntzeff \& Kraft 1996; van Leeuwen et al. 2000). The ADF has a conspicuous peak at $[\mathrm{Fe} / \mathrm{H}] \sim-1.4$ with a long, metal-rich tail starting from $[\mathrm{Fe} / \mathrm{H}] \sim-1$ extending to $[\mathrm{Fe} / \mathrm{H}] \sim-0.5$. It is expected that both the unusual ADF and the peculiar behaviour of n-capture elements could be useful in inferring the potentially unique history of $\omega$ Cen.

\subsection{Star Formation History of $\omega$ Cen}

The star formation history of $\omega$ Cen is inferred from the stellar elemental abundance pattern shown in Figure 5. First of all, a sudden increase in the $[\mathrm{Ba} / \mathrm{Eu}]$ ratios at $[\mathrm{Fe} / \mathrm{H}] \sim-1.6$ implies that $\omega$ Cen has undergone two discrete episodes of star formation. The observed [Ba/Eu] ratios of as large as +1 are attributed to a contribution from asymptotic giant branch (AGB) stars after gas removal at the end of the first episode of star formation. If gas was not expelled after the first episode, the s-process nucleosynthesis in AGB stars would increase the $[\mathrm{Ba} / \mathrm{Eu}]$ ratio to only $\sim+0.2$ at most according to the Salpeter IMF.

The star formation history inferred from the observed $[\mathrm{Ba} / \mathrm{Eu}]$ ratios is then as follows. (a) After completion of the first phase of star formation, a large part of the remaining gas was expelled by SN II explosions. (b) The gas was then contaminated by s-process elements ejected from AGB stars during a quiescent stage lasting several hundred Myr. This led to the enhancement of Ba relative to Eu in the gas. (c) Star formation then recommenced after the gas, once heated by SNe, cooled sufficiently and collapsed, with associated shock wave formation. Stars formed from this shocked gas and stars subsequently formed have very high $[\mathrm{Ba} / \mathrm{Eu}]$ ratios. The second star formation phase continued until the metallicity $[\mathrm{Fe} / \mathrm{H}]$ reached $\sim-1$.

This then poses the question of how star formation proceeded after the second star formation epoch. A clue lies in the extended metal-rich tail in the observed ADF. Pancino et al. (2002) recently found that stars belonging to 
Inhomogeneous Chemical Evolution of the Galactic Halo

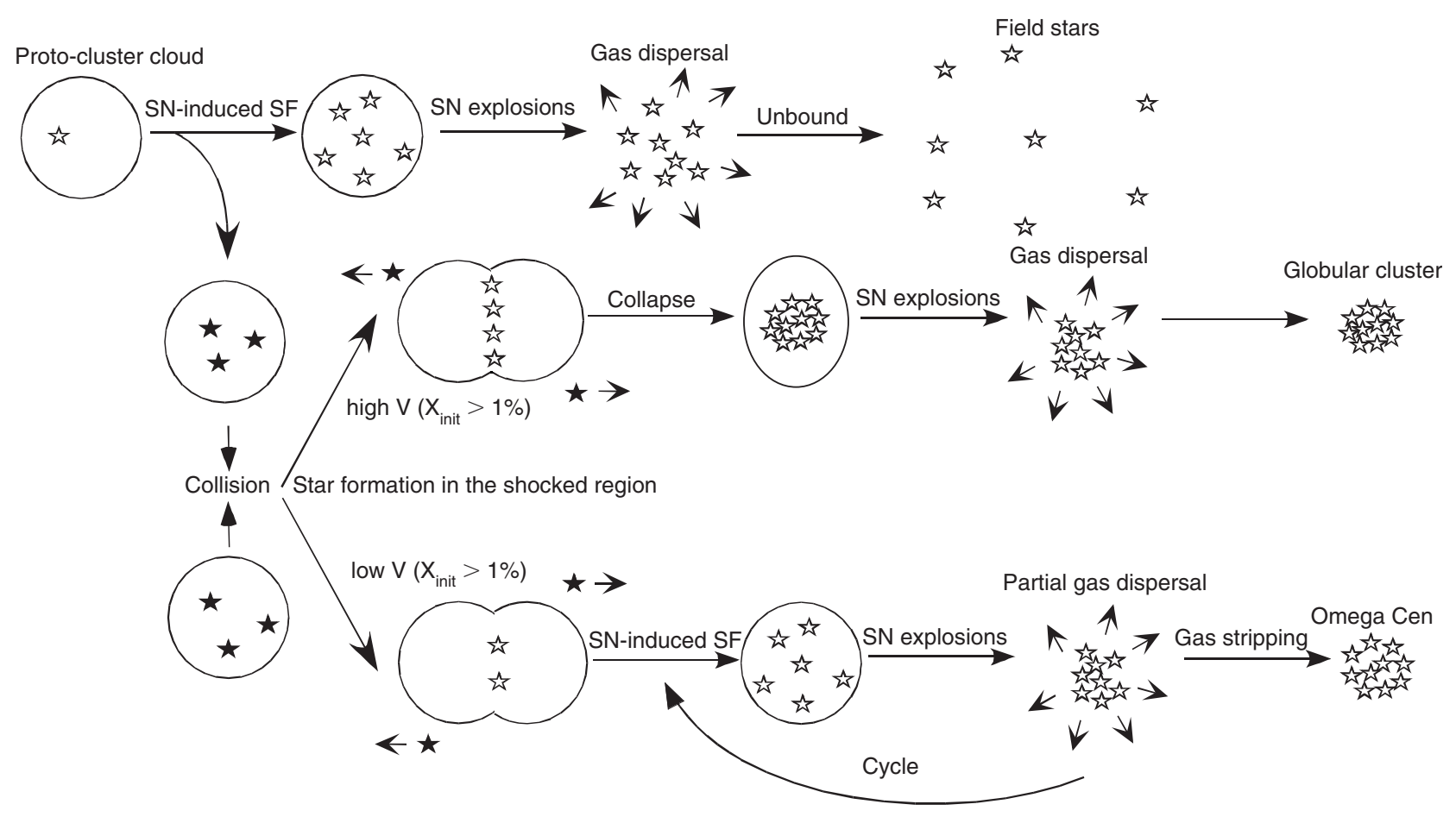

Figure 6 Schematic picture of inhomogeneous chemical evolution model for the Galactic halo.

the metal-rich tail exhibit decreasing $[\mathrm{Ca} / \mathrm{Fe}]$ ratios with increasing $[\mathrm{Fe} / \mathrm{H}]$, ascribed as a sign of SNe Ia enrichment (० in Figure 5). From the theoretical aspect, such SNe Ia enrichment is indispensable to increasing the metallicity $[\mathrm{Fe} / \mathrm{H}]$ of stars to $\sim-0.5$ as observed. In fact, this metalrich end of $[\mathrm{Fe} / \mathrm{H}]$ cannot be reached by the $\mathrm{Fe}$ supply from only SNe II given the small number of stars with $[\mathrm{Fe} / \mathrm{H}]>-1$. In the $\mathrm{SN}$-induced star formation scenario, $\mathrm{SNe}$ Ia do not only enrich gas with $\mathrm{Fe}$ but also initiate star formation in the third episode. In the end, $\omega$ Cen has three episodes of star formation, each of which is likely to be identified with each subpopulation found by Pancino et al. (2000).

\subsection{Globular Cluster Formation Scenario}

The uniqueness of $\omega$ Cen has led some authors to consider that it is the surviving nucleus of a dwarf galaxy that has been tidally stripped by the Milky Way (e.g., Freeman 1993). It should be noted that there are no observed metalpoor $([\mathrm{Fe} / \mathrm{H}] \lesssim-2)$ stars in $\omega$ Cen, which indicates that $\omega$ Cen formed from a pre-enriched gas. This feature is indeed shared with the other GCs. The formation of $\omega$ Cen should therefore be considered in any general scheme of GC formation. Here we propose that GCs formed through collisions of the proto-cluster clouds at the halfway of SNinduced star formation. Cloud-cloud collisions can leave shocked gas with some heavy elements but without stars or dark matter.

After two clouds collide, strong shock waves are formed, which compress the gas and leave a dense cloud with sufficient mass for star formation if the collision is nearly head-on. From this compressed gas, stars are assumed to form with metallicity inherited from the gas. In the SN-driven star formation scenario, the star formation history is determined by the evolution of SNRs, which is affected by the environment. A critical indicator of chemical evolution is whether star formation proceeded for a number of generations or ceased after only one or a few generations. This condition is given by comparing the maximum size that an SNR can attain and the size of the cloud. If the maximum size of an SNR exceeds the size of the cloud, the SNR cannot assemble the gas necessary to form stars of the next generation. The resultant GC would then contain a single generation of stars with no dispersion in $[\mathrm{Fe} / \mathrm{H}]$. If the SNR is smaller than the cloud, the cloud would contain multiple generations of stars with different $[\mathrm{Fe} / \mathrm{H}]$, resembling $\omega$ Cen stars. This criterion gives the maximum velocity that can produce a GC with some dispersion in $[\mathrm{Fe} / \mathrm{H}]$, as follows (see Tsujimoto \& Shigeyama 2003b),

$v_{1, \max } \sim 33 \mathrm{~km} \mathrm{~s}^{-1}\left(\sigma_{v} / 10 \mathrm{~km} \mathrm{~s}^{-1}\right)^{37 / 28}\left(M / 10^{6} \mathrm{M}_{\odot}\right)^{1 / 4}$.

Since clouds move at $\sim 200 \mathrm{~km} \mathrm{~s}^{-1}$ in the gravitational potential of the Milky Way, it is rare that two clouds would collide with such low velocity as long as each cloud has a mass of $\sim 10^{6} \mathrm{M}_{\odot}$ corresponding to the Jeans mass soon after recombination.

An additional condition for the formation of multiple generations of stars is that the mass fraction $X_{\text {init }}$ of stars in a cloud at the beginning should be less than $1 \%$, as discussed previously Tsujimoto et al. (1999). If $X_{\text {init }}$ is 
larger than $1 \%$, the total gas swept up by the first SNRs exceeds the entire amount of available gas, and thus star formation stops at the first or with a few generations.

The mass and velocity dispersion of the proto-cluster cloud generating $\omega$ Cen have been estimated to be $M \sim 3 \times 10^{7} \mathrm{M}_{\odot}$ and $\sigma_{v} \sim 36 \mathrm{~km} \mathrm{~s}^{-1}$ from the chemical evolution model (Tsujimoto \& Shigeyama 2003b). These values together with Equation (2) suggest that a supernova does not break up the shocked clouds if two proto-cluster clouds collide with relative velocity $\left(2 v_{1}\right)$ of less than $\sim 700 \mathrm{~km} \mathrm{~s}^{-1}$. Therefore, $\omega$ Cen-like clusters are most likely to form from two colliding massive clouds if $X_{\text {init }}$ is less than $1 \%$. Such a small $X_{\text {init }}$ could be realised by a small relative velocity leading to weak shock-wave compression. After a small mass fraction $\left(X_{\text {init }}<1 \%\right)$ of first-generation stars had formed in colliding proto-cluster clouds, chemical enrichment due to $\mathrm{SN}$-induced star formation would then have proceeded in $\omega$ Cen. The scheme of GC formation discussed here is described in Figure 6.

\section{Conclusions}

Here we show that stellar elemental abundance patterns for n-capture elements give us a deep insight into the star formation histories for nearby galaxies and $\omega$ Cen. Our finding is brought by two characteristics of n-capture elements; (a) the correlation of $[\mathrm{r}$-process $/ \mathrm{Fe}]$ with $[\mathrm{Fe} / \mathrm{H}]$ in stellar abundances has a quite unique feature as seen in the Galactic halo stars, and (b) two distinct types of n-capture processes, i.e. $r$ - and s-processes, occur in a different site, which yields the time difference in the contribution to the enrichment in the ISM between two processes. In addition, the $[\alpha / \mathrm{Fe}]$ ratio which should represent the SN II/SN Ia ratio and thus serve as a nucleosynthesis clock as well as the s-/r-process elemental ratio is another powerful tool to infer the star formation history, though this might not be the case for $\mathrm{dSph}$ galaxies.

\section{References}

Freeman, K. C. 1993, in ASP Conf. Ser. 48, The Globular ClusterGalaxy Connection, eds. G. H. Smith, \& J. P. Brodie (San Francisco: ASP), 608

Guillot, T. 1999, Planet Space Sci., 47, 1183

McWilliam, A., Preston, G. W., Sneden, C., \& Searle, L. 1995, AJ, 109, 2757

McWilliam, A. 1997, ARA\&A, 35, 503

McWilliam, A. 1998, AJ, 115, 1640

Norris, J. E., \& Da Costa, G. S. 1995, ApJ, 447, 680

Norris, J. E., Freeman, K. C., \& Mighell, K. J. 1996, ApJ, 462, 241

Pancino, E., Ferraro, F. R., Bellazzini, M., Piotto, G., \& Zoccali, M. 2000, ApJ, 534, L83

Pancino, E., Pasquini, L., Hill, V., Ferraro, F. R., \& Bellazzini, M. 2002, ApJ, 568, L101

Pettini, M., Ellison, S. L., Steidel, C. C., Shapley, A. E., \& Bowen, D. V. 2000, ApJ, 532, 65

Prochaska, J. X., \& Wolfe, A. M. 2002, ApJ, 566, 68

Ryan, S. G., Norris, J. E., \& Beers, T. C. 1996, ApJ, 471, 254

Shetrone, M. D., Côté, P., \& Sargent, W. L. 2001, ApJ, 548, 592

Shetrone, M. D., Ven, K. A., Tolstoy, E., Primas, F., Hill, V., \& Kaufer, A. 2003, AJ, 125, 684

Shigeyama, T., \& Tsujimoto, T. 1998, ApJ, 507, L135

Smith, V. V., Suntzeff, N. B., Cunha, K., Gallino, R., Busso, M., Lambert, D. L., \& Straniero, O. 2000, AJ, 119, 1239

Suntzeff, N. B., \& Kraft, R. P. 1996, AJ, 111, 1913

Timmes, F. X., Woosley, S. E., \& Weaver, T. A. 1995, ApJS, 98, 617

Tinsley, B. M. 1980, Fund. Cosmic Phys., 5, 287

Tsujimoto, T., Nomoto, K., Yoshii, Y., Hashimoto, M., Yanagida, S., \& Thielemann, F.-K. 1995, MNRAS, 277, 945

Tsujimoto, T., \& Shigeyama, T. 1998, ApJ, 508, L151

Tsujimoto, T., Shigeyama, T., \& Yoshii, Y. 1999, ApJ, 519, L63

Tsujimoto, T., Shigeyama, T., \& Yoshii, Y. 2000, ApJ, 531, L33

Tsujimoto, T., \& Shigeyama, T. 2001, ApJ, 561, L97

Tsujimoto, T., \& Shigeyama, T. 2002, ApJ, 571, L93

Tsujimoto, T., \& Shigeyama, T. 2003a, Carnegie Observatories Astrophysics Series, Vol. 4: Origin and Evolution of the Elements, eds. A. McWilliam, \& M. Rauch (Pasadena: Carnegie Observatories)

Tsujimoto, T., \& Shigeyama, T. 2003b, ApJ, 590, 803

Welty, D. E., Lauroesch, J. T., Blades, C., Hobbs, L. M., \& York, D. G. 2001, ApJ, 554, L75

Wheeler, J. C., Sneden, C., \& Truran, J. W. 1989, ARA\&A, 27, 279 